\title{
Araucaria Angustifolia: Influence of Mother Tree Sex and Provenance in Grafting Success
}

\author{
Mônica Moreno Gabira ${ }^{1}$ (]) \\ Rosimeri de Oliveira Fragoso ${ }^{2}$ (D) \\ Ivar Wendling ${ }^{3}$ \\ Carlos Andre Stuepp ${ }^{2}$ \\ ${ }^{1}$ Universidade Federal do Paraná (UFPR), Curitiba, PR, Brasil. \\ ${ }^{2}$ Universidade Estadual de Ponta Grossa (UEPG), Ponta Grossa, PR, Brasil. \\ ${ }^{3}$ Empresa Brasileira de Pesquisa Agropecuária (EMBRAPA), Colombo, PR, Brasil.
}

\begin{abstract}
The development of vegetative propagation programs of Araucaria angustifolia still lacks on information about genetic factor that may influence on its success. We aimed to evaluate how mother tree sex and provenance influence on grafting success. So, we collected orthotropic branches from 26 mother trees male and female, of a clonal garden established from matrices from different provenances, and carried out an experiment using a patch grafting technique. Grafting survival, number and length of shoots did not differ between mother tree sex and provenance. The absence of significant variations between mother trees and no correlation with sex or provenance indicates that the morphological characteristics of rootstocks and shoots, and environmental conditions after grafting may have more influence on the success of grafting.
\end{abstract}

Keywords: Clonal silviculture, patch grafting technique, vegetative propagation.

Araucaria angustifolia is the native main species from Mixed Ombrophilous Forest of the Atlantic Forest biome, also known as Araucaria Forest. It is the only species of Araucaria genus occurring naturally in Brazil (Wendling et al., 2017), with expressive economic and ecological importance in its region. Araucaria trees underwent an uncontrolled exploitation during $19^{\text {th }}$ century for wood extraction, which had consequences for its genetic variability and the need of restoration plantings for ecological and commercial purposes (Hess et al., 2018)growth models were fitted with the measures of ring increment data of 587 trees, covering all distribution diameter classes, in four sites in southern Brazil. These fitted models showed the minimum cutting diameter of $40 \mathrm{~cm}$ for about 20 years (maximum annual increment in volume. $A$. angustifolia presents difficulties in the establishment of seed orchards from seedlings due to seed recalcitrant characteristic, production of seedlings with different characteristics from parents (sex, quality of wood and seeds), and a long time to achieve sexual maturity (Rickli-Horst et al., 2019). The fact that araucaria may have significant differences in genetic level between populations makes mother trees provenance another factor of interest (Sousa et al., 2020).

One of the bottlenecks for araucaria use and conservation is the vegetative propagation, specially the production of clonal seedlings (Stuepp et al., 2018). It is known that gymnosperms are very difficult to be propagated by cuttings and the A. angustifolia is not different (Zavattieri et al., 2016); therefore, the use of grafting would be the best option for the establishment of araucaria clonal plantations. However, there is a lack of knowledge on how is the influence of different factors on the success of seedlings production by grafting. Several studies have been developed on vegetative propagation of araucaria (Wendling et al., 2016a; Wendling et al., 2016b; Gaspar et al., 2017; Maggioni et al., 2020), but there are still gaps related to grafting survival and development to be solved for the adequate establishment of commercial plantations. Thus, this study aimed to evaluate the influence of mother tree sex and provenance on survival and initial development of Araucaria angustifolia grafting. 
The experiment was carried out from December/2019 to April/2020 in Ivaí, Paraná, Brazil ( $24^{\circ} 59^{\prime} 16^{\prime \prime}$ S, 50 52 $52^{\prime} 10^{\prime \prime}$ W, $704 \mathrm{~m}$ ). According to the Köppen classification, region's climate is temperate, of the Cfa type, the coldest month temperature between $8{ }^{\circ} \mathrm{C}$ and $21^{\circ} \mathrm{C}$, with well-distributed rains throughout the year and the warmest month temperature below $28^{\circ} \mathrm{C}$ (Alvares et al., 2013).

Araucaria seedlings used as rootstock were produced in $3,780 \mathrm{~cm}^{3}$ pots aged around 18 months. The rootstocks were cleaned at the grafting area around $10.0 \pm 1.0 \mathrm{~cm}$. The shoots of orthotropic branches used as grafts were collected from 26 mother trees male and female, of a clonal garden in Colombo, Paraná, established from matrices (35 years old) from different provenances (Minas Gerais, São Paulo, Paraná, Santa Catarina and Rio Grande do Sul), and rescued by grafting (Table 1). The shoots were packed it in Styrofoam boxes with water and ice until the grafting process.

Table 1. Identification, sex (male and female), and provenance (MG: Minas Gerais; SP: São Paulo; PR: Paraná; SC: Santa Catarina; RS: Rio Grande do Sul), of Araucaria angustifolia mother trees used for grafting.

\begin{tabular}{cccccc} 
Mother tree & Sex & Provenance & Mother tree & Sex & Provenance \\
\hline F4 & Female & SP & F30 & Female & MG \\
F6 & Female & PR & F31 & Female & PR \\
F8 & Female & PR & F33 & Female & PR \\
F12 & Female & SC & F36 & Female & PR \\
F15 & Female & MG & F42 & Female & SC \\
F16 & Female & PR & F44 & Female & MG \\
F17 & Female & PR & M12 & Male & PR \\
F24 & Female & SP & M13 & Male & SP \\
F25 & Female & MG & M17 & Male & Male \\
F26 & Female & PR & M18 & Male & SC \\
F27 & Female & PR & M20 & & SP
\end{tabular}

We used a patch grafting technique, with buds of approximately $4.0 \pm 1.0 \mathrm{~cm}$, which were adjusted to an incision made in the rootstock. Buds were fixed to the rootstock using a $2 \mathrm{~cm}$ wide plastic band until they were welded. After grafting, plants were placed in a shade house (50\% shading) with micro-sprinkler irrigation four times a day for 10 minutes each (144 L hour ${ }^{-1}$ flow rate) for 120 days. After 45 days we removed the plastic bands. We evaluated survival at $60,90,120,150$, and 180 days after grafting. Number of grafts with shoots, number of sprouts/grafts, and sprout length were evaluated at 120,150,180 days after grafting.

The experiment was carried out following a completely randomized design, in a split-plot over time arrangement, with four replications of six grafts per experimental unit, totaling 624 grafts. Data was submitted to the Shapiro-Wilk test to verify the normality of the variables and to Bartlett test to verify homogeneity of variances. A two-way ANOVA was performed and the Tukey test was applied to compare the treatments when ANOVA was significant $(\mathrm{p}<0.05)$.
Our results indicate that there is no relation between the variables and provenance or mother tree sex. Graft survival rates varied significantly, without any relation with provenance or mother tree sex (Figure 1). Grafts from mother trees F36, F29, F4, F31, F28, F12, and F44 had higher survival rates, which were higher than $88 \%$. In the majority of grafts, we did not observe a reduction in survival after 120 days, indicating that these first 120 days after grafting are more important for this variable. Grafts from mother trees F6 and F15 presented the lowest survival rates, with $46 \%$ at 180 days after grafting. Differing from our results, Wendling et al. (2016a) observed higher survival of female grafts in $A$. angustifolia, which was attributed to differences in resources allocation for reproduction. The drastic reduction on survival rates from 60 to 90 days after grafting may be related to plastic bands removal that occurred 45 days after grafting. It may indicate the need of keeping the plastic band for a longer period or changing water management after removing it (Rickli-Horst et al., 2019). 

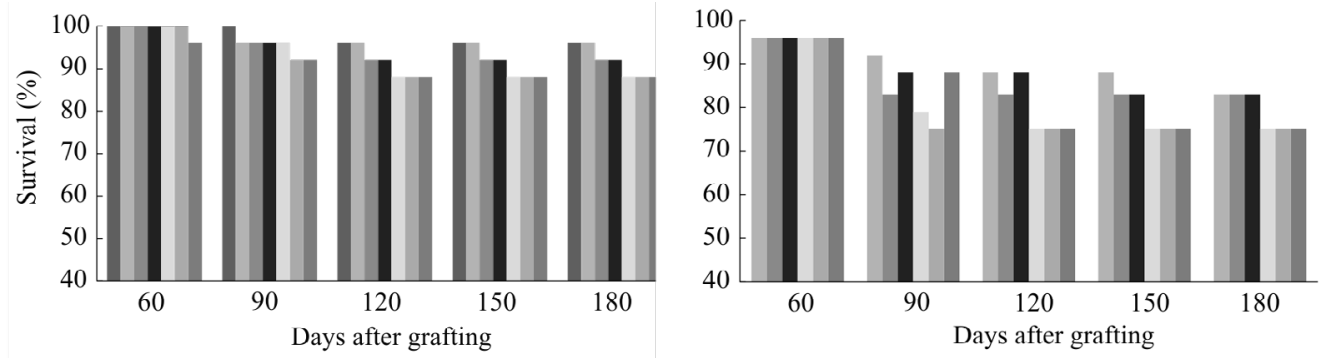

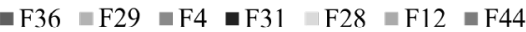

M16 $\quad$ F26 $\quad \mathrm{M} 13 \quad \mathrm{M} 18 \quad \mathrm{~F} 16 \quad \mathrm{~F} 8$
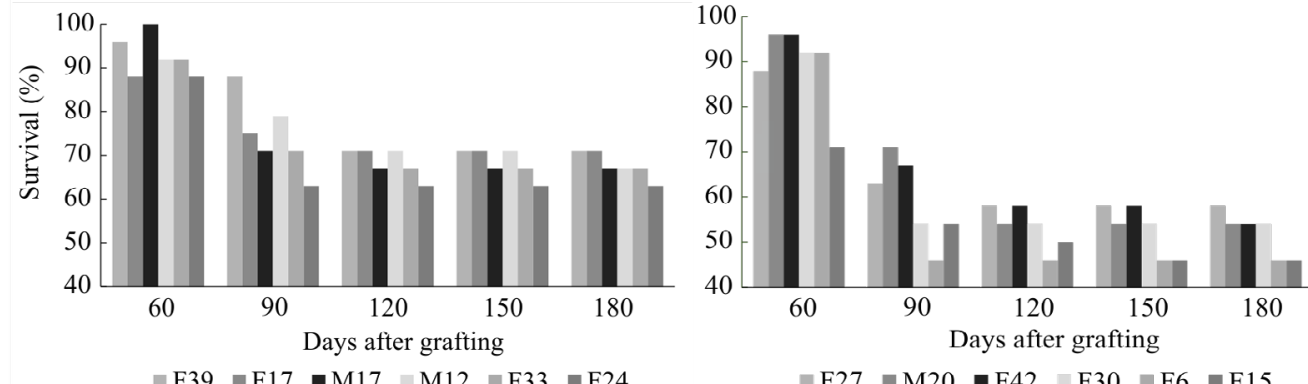

Figure 1. Survival rates of Araucaria angustifolia grafts at 60, 90, 120, 150, and 180 days after grafting.

For the number of grafts with shoots, the number of shoots/ grafts, and shoot length, we observed a high similarity between progenies, with little variation among them (Table 2). Although it did not differ from other grafts, F29 presented higher values for all variables, indicating a high potential to be propagated via grafting technique. We did not observe differences between grafts among evaluation time, indicating that these evaluations could occur only at 180 days, before field planting.

Table 2. Number of grafts with shoots, number of shoots/grafts, and shoot length (cm) of Araucaria angustifolia grafts at 120, 150, and 180 days after grafting. Same letters in columns do not differ from each other by the Tukey test $(\mathrm{p}>0.05)$.

\begin{tabular}{|c|c|c|c|c|c|c|c|c|c|c|c|c|c|c|c|c|c|c|}
\hline \multirow{3}{*}{$\begin{array}{l}\begin{array}{c}\text { Mother } \\
\text { tree }\end{array} \\
\text { F29 }\end{array}$} & \multicolumn{6}{|c|}{ Number of grafts with shoots } & \multicolumn{6}{|c|}{ Number of shoots/grafts } & \multicolumn{6}{|c|}{ Shoot length $(\mathrm{cm})$} \\
\hline & \multicolumn{2}{|c|}{ 120D } & \multicolumn{2}{|c|}{$150 \mathrm{D}$} & \multicolumn{2}{|c|}{$180 \mathrm{D}$} & \multicolumn{2}{|c|}{ 120D } & \multicolumn{2}{|c|}{ 150D } & \multicolumn{2}{|c|}{ 180D } & \multicolumn{2}{|c|}{ 120D } & \multicolumn{2}{|c|}{ 150D } & \multicolumn{2}{|c|}{ 180D } \\
\hline & 0.88 & $\mathrm{a}$ & 0.88 & $\mathrm{a}$ & 0.92 & $\mathrm{a}$ & 6.50 & $\mathrm{a}$ & 7.25 & $\mathrm{a}$ & 8.75 & $\mathrm{a}$ & 4.25 & a & 6.13 & $\mathrm{a}$ & 7.63 & a \\
\hline F28 & 0.54 & $\mathrm{ab}$ & 0.58 & $\mathrm{ab}$ & 0.67 & $\mathrm{ab}$ & 4.00 & $a b$ & 4.25 & $\mathrm{ab}$ & 4.75 & $\mathrm{ab}$ & 3.38 & $\mathrm{a}$ & 5.13 & $\mathrm{a}$ & 6.50 & $\mathrm{ab}$ \\
\hline F12 & 0.50 & $\mathrm{ab}$ & 0.50 & $a b$ & 0.63 & $a b$ & 3.00 & $\mathrm{ab}$ & 3.00 & $\mathrm{ab}$ & 4.25 & $a b$ & 1.00 & a & 1.13 & $\mathrm{a}$ & 1.88 & $a b$ \\
\hline F25 & 0.54 & $\mathrm{ab}$ & 0.54 & $\mathrm{ab}$ & 0.58 & $a b$ & 3.75 & $a b$ & 3.75 & $a b$ & 4.00 & $a b$ & 0.80 & $\mathrm{a}$ & 1.45 & $\mathrm{a}$ & 2.85 & $a b$ \\
\hline F36 & 0.46 & $a b$ & 0.46 & $a b$ & 0.54 & $a b$ & 2.75 & $a b$ & 2.75 & $a b$ & 3.25 & $\mathrm{~b}$ & 1.63 & $\mathrm{a}$ & 2.75 & a & 3.00 & $a b$ \\
\hline M13 & 0.46 & $a b$ & 0.46 & $a b$ & 0.50 & $a b$ & 3.00 & $a b$ & 3.00 & $a b$ & 3.25 & $\mathrm{~b}$ & 3.88 & $\mathrm{a}$ & 4.13 & $\mathrm{a}$ & 5.00 & $a b$ \\
\hline M12 & 0.46 & $a b$ & 0.46 & $a b$ & 0.46 & $a b$ & 3.00 & $a b$ & 3.00 & $a b$ & 3.25 & $\mathrm{~b}$ & 1.38 & a & 3.13 & $\mathrm{a}$ & 4.25 & $a b$ \\
\hline F31 & 0.42 & $a b$ & 0.54 & $\mathrm{ab}$ & 0.58 & $a b$ & 2.50 & $a b$ & 3.25 & $a b$ & 3.50 & $a b$ & 1.08 & $\mathrm{a}$ & 1.88 & $\mathrm{a}$ & 1.88 & $a b$ \\
\hline M16 & 0.38 & $a b$ & 0.46 & $a b$ & 0.58 & $a b$ & 2.25 & $a b$ & 3.00 & $a b$ & 3.75 & $a b$ & 2.00 & $\mathrm{a}$ & 3.25 & $\mathrm{a}$ & 3.63 & $a b$ \\
\hline F15 & 0.38 & $a b$ & 0.38 & $\mathrm{ab}$ & 0.42 & $a b$ & 2.75 & $a b$ & 2.75 & $\mathrm{ab}$ & 3.00 & $\mathrm{~b}$ & 1.75 & $\mathrm{a}$ & 2.38 & $\mathrm{a}$ & 2.50 & $\mathrm{ab}$ \\
\hline F42 & 0.33 & $a b$ & 0.33 & $a b$ & 0.38 & $a b$ & 2.25 & $a b$ & 2.25 & $a b$ & 2.50 & $\mathrm{~b}$ & 3.00 & a & 3.13 & $a$ & 3.88 & $\mathrm{ab}$ \\
\hline F16 & 0.29 & $a b$ & 0.42 & $\mathrm{ab}$ & 0.42 & $a b$ & 1.75 & $a b$ & 2.50 & $a b$ & 2.50 & $\mathrm{~b}$ & 2.75 & $\mathrm{a}$ & 4.00 & $\mathrm{a}$ & 4.50 & $a b$ \\
\hline F26 & 0.25 & $a b$ & 0.42 & $a b$ & 0.42 & $a b$ & 1.50 & $\mathrm{~b}$ & 2.50 & $a b$ & 2.75 & $\mathrm{~b}$ & 1.75 & $\mathrm{a}$ & 2.50 & $a$ & 2.88 & $\mathrm{ab}$ \\
\hline F39 & 0.29 & $a b$ & 0.33 & $\mathrm{ab}$ & 0.33 & $\mathrm{~b}$ & 1.75 & $a b$ & 2.50 & $a b$ & 2.50 & $\mathrm{~b}$ & 0.68 & $\mathrm{a}$ & 0.73 & $\mathrm{a}$ & 1.18 & $a b$ \\
\hline F4 & 0.13 & $\mathrm{~b}$ & 0.17 & $\mathrm{~b}$ & 0.33 & $\mathrm{~b}$ & 0.75 & $\mathrm{~b}$ & 1.00 & $\mathrm{~b}$ & 2.00 & $\mathrm{~b}$ & 0.63 & $\mathrm{a}$ & 0.88 & $\mathrm{a}$ & 1.68 & $\mathrm{ab}$ \\
\hline F6 & 0.21 & $\mathrm{~b}$ & 0.33 & $a b$ & 0.38 & $a b$ & 1.25 & $\mathrm{~b}$ & 2.00 & $\mathrm{~b}$ & 2.50 & $\mathrm{~b}$ & 1.50 & $\mathrm{a}$ & 2.30 & $\mathrm{a}$ & 4.13 & $\mathrm{ab}$ \\
\hline F30 & 0.29 & $a b$ & 0.29 & $a b$ & 0.29 & $\mathrm{~b}$ & 1.75 & $a b$ & 2.75 & $a b$ & 2.75 & $\mathrm{~b}$ & 0.78 & $\mathrm{a}$ & 1.05 & $\mathrm{a}$ & 1.50 & $\mathrm{ab}$ \\
\hline M20 & 0.25 & $\mathrm{ab}$ & 0.25 & $\mathrm{~b}$ & 0.33 & $\mathrm{~b}$ & 1.50 & $\mathrm{~b}$ & 1.50 & $\mathrm{~b}$ & 2.00 & $\mathrm{~b}$ & 3.00 & $\mathrm{a}$ & 3.63 & $\mathrm{a}$ & 3.68 & $a b$ \\
\hline F27 & 0.21 & $\mathrm{~b}$ & 0.21 & b & 0.21 & $\mathrm{~b}$ & 1.75 & $a b$ & 1.75 & $\mathrm{~b}$ & 1.75 & $\mathrm{~b}$ & 2.88 & $\mathrm{a}$ & 3.25 & $a$ & 4.00 & $\mathrm{ab}$ \\
\hline F33 & 0.17 & $\mathrm{~b}$ & 0.25 & b & 0.25 & $\mathrm{~b}$ & 1.25 & $\mathrm{~b}$ & 2.00 & $\mathrm{~b}$ & 2.00 & $\mathrm{~b}$ & 2.25 & $\mathrm{a}$ & 2.88 & $\mathrm{a}$ & 2.88 & $a b$ \\
\hline M18 & 0.13 & $\mathrm{~b}$ & 0.17 & $\mathrm{~b}$ & 0.17 & $\mathrm{~b}$ & 1.00 & $\mathrm{~b}$ & 1.25 & $\mathrm{~b}$ & 1.00 & $\mathrm{~b}$ & 0.25 & $\mathrm{a}$ & 0.50 & $\mathrm{a}$ & 0.50 & b \\
\hline F17 & 0.08 & $\mathrm{~b}$ & 0.21 & $\mathrm{~b}$ & 0.38 & $a b$ & 0.50 & $\mathrm{~b}$ & 1.25 & $\mathrm{~b}$ & 2.25 & $\mathrm{~b}$ & 0.63 & $\mathrm{a}$ & 1.93 & $\mathrm{a}$ & 2.05 & $a b$ \\
\hline F24 & 0.04 & $\mathrm{~b}$ & 0.13 & $\mathrm{~b}$ & 0.13 & $\mathrm{~b}$ & 0.25 & $\mathrm{~b}$ & 0.75 & $\mathrm{~b}$ & 0.75 & $\mathrm{~b}$ & 0.03 & $\mathrm{a}$ & 0.10 & $a$ & 0.30 & b \\
\hline M17 & 0.04 & $\mathrm{~b}$ & 0.13 & $\mathrm{~b}$ & 0.13 & $\mathrm{~b}$ & 0.25 & $\mathrm{~b}$ & 1.00 & $\mathrm{~b}$ & 1.00 & $\mathrm{~b}$ & 0.03 & $\mathrm{a}$ & 0.18 & $\mathrm{a}$ & 0.68 & $\mathrm{~b}$ \\
\hline F44 & 0.04 & b & 0.08 & b & 0.08 & b & 0.25 & $\mathrm{~b}$ & 0.50 & b & 0.50 & $\mathrm{~b}$ & 0.03 & $\mathrm{a}$ & 0.18 & $\mathrm{a}$ & 0.00 & b \\
\hline F8 & 0.00 & b & 0.04 & $\mathrm{~b}$ & 0.13 & $\mathrm{~b}$ & 0.00 & $\mathrm{~b}$ & 0.25 & $\mathrm{~b}$ & 0.75 & $\mathrm{~b}$ & 0.00 & $\mathrm{a}$ & 0.05 & $\mathrm{a}$ & 0.10 & b \\
\hline
\end{tabular}


Sousa et al. (2020) identified two distinct populations of A. angustifolia in South and Southern Brazil, highlighting the importance of genetic and environmental factors on their evolutionary history. The lack of differences among provenance of mother trees on the success of grafting evidence the potential of rescuing several genetic materials, which would critical for species genetic conservation.

This study confirms the potential of propagating A. angustifolia by grafting, although the lack of knowledge about genetic variation and grafts provenance difficult more specifical propagation programs. The absence of significant variations between the mother trees we used in this study and no correlation with sex or provenance indicates that factors such as morphological characteristics of rootstocks and shoots, as observed by Stuepp et al. (2020), and environmental conditions after grafting may have more influence on the success of grafting.

\section{ACKNOWLEDGEMENTS}

To Bitumirim Ind. e Com. de Erva-Mate Ltda and to the Brazilian Agricultural Research Corporation - Embrapa.

\section{SUBMISSION STATUS}

Received: 4 Oct. 2021

Accepted: 25 Nov. 2021

Associate editor: José Carlos Arthur Junior

\section{CORRESPONDENCE TO}

\section{Rosimeri de Oliveira Fragoso}

Universidade Estadual de Ponta Grossa, Avenida General Carlos Cavalcanti, 4748, Ponta Grossa, PR, Brasil.

e-mail: meri_ol@yahoo.com.br

\section{AUTHORS' CONTRIBUTIONS}

Mônica Moreno Gabira: formal analysis (equal); writing original draft (equal); writing - review \& editing (equal).

Rosimeri de Oliveira Fragoso: formal analysis (equal); writing - review \& editing (equal).

Ivar Wendling: formal analysis (equal); methodology (equal); writing - review \& editing (equal).

Carlos Andre Stuepp: formal analysis (equal); methodology (equal); project administration (equal); Writing - review \& editing (equal).

\section{REFERENCES}

Alvares CA, Stape JL, Sentelhas PC, Gonçalves JLM, Sparovek G. Köppen's Climate Classification Map for Brazil. Meteorologische Zeitschrift 2013, 22(6): 711-728.

Gaspar RGB, Wendling I, Stuepp CA, Angelo AC. Rootstock age and growth habit influence top grafting in Araucaria Angustifolia. Cerne 2017, 23(4): 465-471.

Hess AF, Loiola T, Souza IA, Minatti M, Ricken P, Borsoi GA. Forest management for the conservation of Araucaria Angustifolia in Southern Brazil. Floresta 2018, 48(3): 373-382.

Maggioni RA, Gabira MM, Wedling I, Zuffellato-Ribas KC. Giberelina, sombreamento e altura de poda na produção de brotações epicórmicas em Araucaria Angustifolia (Bertol.). Revista Forestal Mesoamericana Kurú 2021, 18(42): 30-36.

Rickli-Horst HC, Wendling I, Koehler HS, Zuffellato-Ribas KC. Araucaria Angustifolia budding techniques in indoor and outdoor stablished rootstocks. Floresta e Ambiente 2019, 26(3): E20170799.

Sousa VA, Reeves PA, Reilley A, Aguiar AV, Stefenon VM, Richards CM. Genetic diversity and biogeographic determinants of population structure in Araucaria Angustifolia (Bert.) O. Ktze. Conservation Genetics 2020, 21: 217-229.

Stuepp CA, Paula FA, Cordeiro EC, Santos ECC, Gabira MM, Wendling I. New technique for the early production of Araucaria angustifolia grafted plants. Pesquisa Agropecuária Brasileira 2020, 55: e02030.

Stuepp CA, Wendling I, Xavier A, Zuffellato-Ribas KC. Vegetative propagation and application of clonal forestry in brazilian native tree species. Pesquisa Agropecuária Brasileira 2018, 53(9): 9851002 .

Wendling I, Stuepp CA, Zuffellato-Ribas KC. Araucaria clonal forestry: types of cuttings and mother tree sex in field survival and growth. Cerne 2016a, 22(1): 19-26.

Wendling I, Stuepp CA, Zuffellato-Ribas KC. Araucaria Angustifolia grafting: techniques, environments and origin of propragation material. Bosque 2016b, 37(2): 285-293.

Wendling I, Zanette F, Rickli-Horst, HC, Constantino V. Produção de mudas de A. Angustifolia por enxertia. In: Wendling I, Zanette F, editors. Araucária: particularidades, propagação e manejo de plantios. Brasilia: Embrapa, 2017.

Zavattieri MA, Ragonezi C, Klimaszewska K. Adventitious rooting of conifers: influence of biological factors. Trees 2016, 30: 1021-1032. 